Ambiente \& Água - An Interdisciplinary Journal of Applied Science
ISSN 1980-993X - doi:10.4136/1980-993X
www.ambi-agua.net
E-mail: ambi.agua@gmail.com

\title{
Sustentabilidade de cardápio: avaliação da pegada hídrica nas refeições de um restaurante universitário
}

\author{
doi:10.4136/ambi-agua.1664 \\ Received: 18 May 2015; Accepted: 10 Sep. 2015 \\ Virgílio José Strasburg ${ }^{1}$; Vanusca Dalosto Jahno ${ }^{2}$ \\ ${ }^{1}$ Universidade Federal do Rio Grande do Sul (UFRGS), Porto Alegre, RS, Brasil \\ Departamento de Nutrição. Faculdade de Medicina. Cesan/HCPA \\ ${ }^{2}$ Universidade Feevale.Novo Hamburgo, RS, Brasil \\ Programa de Pós-Graduação em Qualidade Ambiental (PPGQA) \\ *Autor correspondente: e-mail: vjs.nut@terra.com.br, \\ vanusca@feevale.br
}

\section{RESUMO}

A água é um recurso fundamental para a produção de alimentos e também de refeições. No presente estudo, a pegada hídrica $(\mathrm{PH})$ na composição de cardápios composto por cereal, leguminosa, carne, guarnição, salada e sobremesa foi avaliada no período de uma quinzena em um restaurante universitário público na cidade de Porto Alegre / RS. A realização da coleta de dados ocorreu no período de 2 a 13 de setembro de 2013. Foi feito o levantamento do cardápio quinzenal com as respectivas quantidades de alimentos utilizados, provisionados para 1800 refeições (almoço e jantar). As quantidades da $\mathrm{PH}$ por alimento foram estimadas com base na literatura científica. Os dados coletados foram calculados em frequências absolutas, em percentual e por média. Foi constatado que todos os produtos de origem vegetal composto por cereais, leguminosas, verduras e frutas corresponderam a $65,5 \%$ do total de matéria prima, mas contribuem com apenas $22,1 \%$ da $\mathrm{PH}$ do cardápio. Os produtos de origem animal (ovos, carnes bovina e de frango) representaram 77,9\% da PH. Quanto à avaliação da PH nos cardápios foi identificada uma média diária per capita de 2099,1 litros por refeição, sendo que o item carne bovina foi o que mais contribuiu nesse resultado. Na fase de planejamento de cardápios, a avaliação da pegada hídrica em refeições é uma das possibilidades para a sustentabilidade dos recursos naturais do planeta e para a formação de hábitos e consumo de alimentos.

Palavras-chave: ecologia, indicadores ambientais, uso da água.

\section{Menu of sustainability: evaluation of water footprint in a university dining restaurant}

\begin{abstract}
Water is a key resource for the production of food and meals. This study evaluated the water footprint (WF) of menus consisting of cereal, legumes, meat, garnish, salad and dessert at a public university in the city of Porto Alegre / RS. Data collection took place between September 2 and 13, 2013. The biweekly menu was assessed to verify the amount of food
\end{abstract}


used for making 1800 meals (lunch and dinner). The size of the food's water footprint was estimated based upon the scientific literature. The data were calculated in absolute frequencies, in percentages and averages. It was found that vegetable products (cereals, legumes, vegetables, and fruits) represented $65.5 \%$ of the total raw material in kilograms, but were responsible for only $22.1 \%$ of the total WF. Animal products (eggs, meat and chicken) were responsible for $77.9 \%$ of the WF. This assessment of the WF of menus identified a daily average per capita of 2099.1 litres per meal, and meat was the largest contributor to this result. Evaluating the water footprint of foods used in menu planning fosters the sustainability of the planet's natural resources and encourages new habits and methods of sustainable food consumption.

Keywords: ecology, environmental indicators, water use.

\section{INTRODUÇÃO}

O conceito de sustentabilidade tem ganhado destaque sobre a forma como o ser humano tem se utilizado dos recursos naturais. $\mathrm{O}$ surgimento de termos como pegadas ecológica, hídrica e de carbono demonstra que a humanidade está vivendo atualmente além da capacidade do planeta (Galli et al., 2012). A água está presente em todos os processos e atividades humanas, dentre os quais os relacionados com a produção de alimentos, tanto para a produção agrícola como pecuária, sendo componente indispensável para garantir a capacidade de produção. Da mesma forma, no beneficiamento dos alimentos entra nos processos industriais de forma direta com as matérias primas e também para o funcionamento dos equipamentos utilizados (Vanham e Bidoglio, 2013). Em relação à água, a utilização de forma racional desse recurso tem sido assunto de pauta frente ao desafio de sua disponibilidade para a sobrevivência dos habitantes da Terra. Diante da necessidade do equilíbrio entre as ações do ser humano na natureza são criados indicadores para avaliação do uso de recursos com vistas à sustentabilidade (Ercin et al., 2011). No âmbito da água, surge o conceito da pegada hídrica (PH) elaborado pelos pesquisadores Hoekstra e Haung (2002) da University of Twente na Holanda. Dentro do conceito de PH surge ainda o termo água virtual (AV). O termo "virtual" diz respeito ao fato de que a maioria da água usada para produzir um produto não está contida nele (Silva et al., 2013a). A mensuração do volume de água doce envolvida ao longo das várias fases do processo produtivo de qualquer produto (mercadoria, bem ou serviço) industrial ou agrícola passa a ser denominado de água virtual (Hoekstra e Chapagain, 2007). Aldaya e Hoekstra (2010) argumentam que a AV pode ser compreendida como uma fonte alternativa de água. Regiões e países com baixa disponibilidade hídrica tem se utilizado desse recurso para garantir o abastecimento de produtos agrícolas para seus habitantes. O Brasil é um grande produtor mundial de alimentos e também considerado como um dos grandes exportadores de AV por meio de suas commodities (Carmo et al., 2007). Gerbens-Leenes e Hoekstra (2012) definem a pegada hídrica como uma medida volumétrica que mostra o consumo de água doce (em metros cúbicos por ano) alocadas no tempo e no espaço. Para Hoekstra e Chapagain (2008) o conceito de PH incorpora implicações quanto ao uso da água relacionado à sua escassez, dependência, seu uso racional e sustentável, bem como de implicações de uma gestão global. A PH pode ser estimada num contexto individual ou coletivo considerando o cálculo da AV contido em cada um dos bens ou serviços consumidos por uma família, ou espaço geográfico como cidade, região ou país. Existe uma estreita relação entre AV e a PH (Chapagain e Orr, 2009). Conceitualmente se define a PH como o volume direto e indireto utilizado de água nos processos que compreendem da produção ao consumo de um bem ou serviço ao longo da cadeia produtiva (Yu et al., 2010). O somatório da PH contempla três componentes: as águas azul, verde e cinza. A água azul 
corresponde à água doce superficial e/ou subterrânea. A água verde é definida como sendo a água oriunda de precipitações e a cinza é o resultado que indica o grau de poluição de água doce associada a um processo produtivo (Hoekstra, 2011). O conteúdo de AV estimado nos produtos utilizados ou consumidos individual ou coletivamente deve ser considerado na estimativa de PH. No entanto Silva et al. (2013a) consideram como incipientes os estudos sobre a PH no país. Estudos considerando a PH como método de análise feito por pesquisadores brasileiros abordam as áreas de produção animal (Palhares, 2011; 2012; 2014), cosméticos (Francke e Castro, 2013), de celulose (Empinotti et al., 2013) na análise de padrões de consumo em dietas (Silva et al., 2013a). E também na relação da PH com o ambiente (Sinisgalli e Tadeu, 2012) e na análise do método da pegada hídrica na governança da água (Empinotti e Jacobi, 2013). O acesso à alimentação é um direito universal, e essa deve promover a saúde, respeitar a diversidade cultural e ser social, econômica e ambientalmente sustentável (Fagury, 2010). O uso dos alimentos é condição básica para o fornecimento de refeições para coletividades. No Brasil, o mercado de refeições para coletividades está em constante crescimento alcançando 19,5 milhões de refeições ao dia no ano de 2014 (Aberc, 2015). No segmento de produção de refeições para coletividades ocorrem uma série de atividades que causam impactos ambientais. Sob o aspecto da sustentabilidade, nesse segmento os estudos brasileiros que vem sendo realizados costumam estar relacionados com a gestão de resíduos sólidos e desperdício de alimentos em etapas de preparo e consumo (Pedro e Claro, 2010; Soares et al., 2011; Collares e Figueiredo, 2012; Barthichoto et al., 2013; Strasburg e Passos, 2014). Considerando a importância de explorar o aspecto da pegada hídrica como elemento de sustentabilidade ambiental essa pesquisa se justifica frente à relevância da necessidade de se investigar sobre os aspectos de fornecimento de refeições num contexto de coletividade. Nesse âmbito, esse estudo teve por objetivo realizar uma investigação sobre a pegada hídrica dos insumos utilizados na composição dos cardápios fornecidos por uma quinzena em um restaurante institucional universitário na cidade de Porto Alegre (RS).

\section{MATERIAIS E MÉTODOS}

Este trabalho é caracterizado como um estudo de caso e foi realizado em um restaurante universitário (RU) de uma universidade pública federal na cidade de Porto Alegre / RS. A realização da coleta de dados ocorreu de 2 a 6 e de 9 a 13 de setembro de 2013. Neste estudo foi aplicado um método experimental piloto para calcular a $\mathrm{PH}$ dos insumos utilizados e escolhido um período amostral por conveniência. Outra justificativa para o período é que pelo fato do local de estudo estar vinculado ao serviço público, a aquisição dos itens utilizados fica limitado a trâmites burocráticos relacionados com licitações. Nesse período foram seguidas as etapas descritas a seguir. A composição de cada cardápio diário foi verificada com a respectiva requisição contendo os ingredientes e quantidades necessárias para o atendimento de uma previsão diária de 1800 refeições para os serviços de almoço e jantar. Essa constante (1800) foi utilizada para calcular o per capita da pegada hídrica $(\mathrm{PH})$ de cada cardápio diário, sendo este composto por cereal (arroz), leguminosa (feijão ou lentilha), prato proteico (de origem animal), guarnição, salada e sobremesa (fruta). Posteriormente foi elaborado um quadro contendo a relação de todos os alimentos com a respectiva $\mathrm{PH}$ utilizados no cardápio do RU. Para o levantamento dessa informação foram utilizados como referência os dados dos estudos de Hoekstra (2010) para os produtos de origem animal e de Mekonnen e Hoekstra (2011) para os produtos de origem vegetal. A organização dessas informações é apresentada na Tabela 1. 
Tabela 1. Relação de alimentos de referência para os cálculos de PH em cardápio de RU de Porto Alegre /RS. Setembro de 2013.

\begin{tabular}{|c|c|c|c|c|c|}
\hline Alimento & Unid. & $\mathrm{PH}\left(\mathrm{L} \mathrm{kg}^{-1}\right)$ & Alimento & Unid. & $\mathrm{PH}\left(\mathrm{L} \mathrm{kg}^{-1}\right)$ \\
\hline Feijão preto & $\mathrm{kg}$ & 5053 & Arroz branco & $\mathrm{kg}$ & 2497 \\
\hline Lentilha & $\mathrm{kg}$ & 5874 & Farinha de mandioca & $\mathrm{kg}$ & 1878 \\
\hline Óleo de soja & $\mathrm{L}$ & 4190 & Farinha de milho & $\mathrm{kg}$ & 1253 \\
\hline Carne bovina & $\mathrm{kg}$ & 15500 & Farinha de trigo & $\mathrm{kg}$ & 1849 \\
\hline Frango & $\mathrm{kg}$ & 3900 & Alface & $\mathrm{kg}$ & 237 \\
\hline Ovo & $\mathrm{kg}$ & 3300 & Agrião (*) & $\mathrm{kg}$ & 237 \\
\hline Linguiça (suína) $\left(^{*}\right)$ & $\mathrm{kg}$ & 4800 & Chicória (*) & $\mathrm{kg}$ & 237 \\
\hline Presunto $(*)$ & $\mathrm{kg}$ & 4800 & Acelga $(*)$ & $\mathrm{kg}$ & 237 \\
\hline Queijo & $\mathrm{kg}$ & 5000 & Rúcula (*) & $\mathrm{kg}$ & 237 \\
\hline Abacaxi & $\mathrm{kg}$ & 255 & Batata doce & $\mathrm{kg}$ & 383 \\
\hline Banana (prata) & $\mathrm{kg}$ & 790 & Cebola & $\mathrm{kg}$ & 345 \\
\hline Laranja (pêra) & $\mathrm{kg}$ & 560 & Pimentão & $\mathrm{kg}$ & 7611 \\
\hline Maçã (fuji) & $\mathrm{kg}$ & 822 & Repolho & $\mathrm{kg}$ & 280 \\
\hline Mamão (formosa) & $\mathrm{kg}$ & 460 & Tomate & $\mathrm{kg}$ & 214 \\
\hline Melão (*) & $\mathrm{kg}$ & 235 & Vagem & $\mathrm{kg}$ & 561 \\
\hline
\end{tabular}

Fonte: adaptado de Hekonnen e Hoekstra (2011) e Hoekstra (2011).

Legenda: Unid: unidade de medida; kg: quilograma; L: litro; PH L kg${ }^{-1}$ : Pegada Hídrica (em litros) por unidade de medida. (*) adaptação feita pelos autores.

Esses valores de referência de $\mathrm{PH}$ utilizados pelos autores são estimativas das médias mundiais e dessa forma isso pode ser considerado como uma limitação. No entanto, essas publicações possibilitam uma quantidade maior de alimentos e permitem sistematizar melhor as informações. De acordo com Carmo et al. (2007) o cálculo de PH de cada produto pode apresentar variações em função de características regionais específicas, como solo e clima. Palhares (2012) argumenta ainda, que qualquer que seja o cálculo de uma pegada (hídrica, ecológica ou de carbono) irá expressar tendências e não valores exatos. Tendo esse quadro de referência pronto, a etapa seguinte foi avaliar todas as preparações diárias do cardápio. Para isso foi desenvolvida uma planilha eletrônica para fazer o cálculo per capita da pegada hídrica na refeição do dia, conforme exemplo da Tabela 2.

Tabela 2. Formulário desenvolvido para calcular a PH per capita do cardápio em RU de Porto Alegre / RS. Setembro/2013.

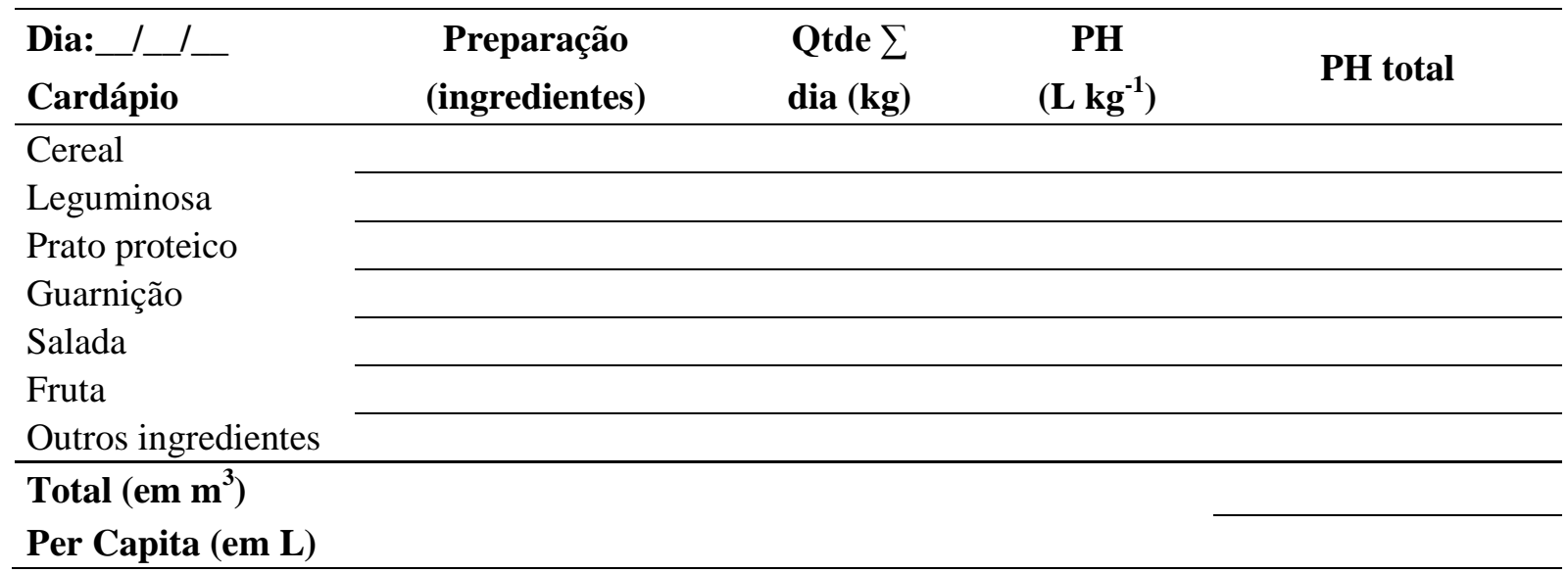

Fonte: elaborado pelo autor.

Legenda: Qtde: quantidade; $\sum$ : somatório; kg: quilograma; $\mathrm{PH} \mathrm{L} \mathrm{kg}{ }^{-1}$ : Pegada Hídrica (em litros). 
O resultado da PH total foi obtido multiplicando os valores das colunas Qtde $\sum$ dia $(\mathrm{kg})$ e PH L $\mathrm{kg}^{-1}$ para cada tipo de alimento. Na realização do cálculo de cada preparação foram considerados os valores da $\mathrm{PH}$ dos alimentos in natura. Não foi considerada nos cálculos a adição de água para a realização das preparações denominada de fator de cocção (Fcy) que define o rendimento do alimento preparado (Ornellas, 2007).

Com todas as informações coletadas, foi possível desenvolver as relações de avaliação do total da PH per capita de todos os cardápios servidos. Da mesma forma foi possível verificar a contribuição de cada grupo alimentar em relação à $\mathrm{PH}$ total do cardápio conforme será apresentado nos resultados.

As informações coletadas foram calculadas em frequência absoluta, em percentual e por média no software Excel versão $97-2003^{\circledR}$. Este estudo fez parte de projeto de pesquisa cadastrado junto a Comissão de Pesquisa da Faculdade de Medicina da Universidade Federal do Rio Grande do Sul sob o registro n 22477/2012.

\section{RESULTADOS E DISCUSSÃO}

A descrição das preparações foi o primeiro passo para identificar os diversos os alimentos constituintes do cardápio e os valores da PH. A Tabela 3 apresenta a descrição das preparações servidas no restaurante universitário.

Tabela 3. Preparações do cardápio de RU de Porto Alegre / RS. Setembro de 2013.

\begin{tabular}{|c|c|}
\hline Itens do Cardápio & Preparações (frequência) \\
\hline Cereal & $\operatorname{arroz}$ branco $(10 \mathrm{x})$. \\
\hline Leguminosa & feijão preto $(8 \mathrm{x})$; feijão carioca e lentilha ( $1 \mathrm{x}$ cada). \\
\hline Prato protéico & $\begin{array}{l}\text { frango: cubos; bife; risoto e sobrecoxa ( } 1 \text { x cada). } \\
\text { bovina: bife }(3 \mathrm{x}) \text {; lagarto* }(2 \mathrm{x}) \text {; e espetinho }(1 \mathrm{x}) \text {. }\end{array}$ \\
\hline Guarnição & $\begin{array}{l}\text { batata doce; cless**; creme de ervilhas; farofa salgada; omelete; polenta; } \\
\text { salada de feijão fradinho; salpicão; torta de vagem; e vagem com ovos } \\
\text { (1 x cada). }\end{array}$ \\
\hline Salada & $\begin{array}{l}\text { acelga; alface; e rúcula ( } 2 \text { x cada); agrião; almeirão; chicória; e tricolor*** } \\
\text { ( } 1 \text { x cada). }\end{array}$ \\
\hline Sobremesa & abacaxi; banana; laranja; mamão; e melão ( 2 x cada). \\
\hline
\end{tabular}

Legenda: $x$ : vezes;

Obs.: * lagarto (corte bovino);

** cless (massa feita com farinha de trigo, ovos e água);

*** tricolor (salada preparada com repolho, cebola e tomate).

Todos os alimentos utilizados foram quantificados para se avaliar a representatividade em kg e da PH em cada grupo de alimentos. A Figura 1 apresenta o resultado dessa avaliação.

Avaliando a distribuição dos grupos de alimentos foi possível constatar que todos os produtos de origem vegetal (cereais, leguminosas, verduras, legumes e frutas) forneceram $65,5 \%$ do total em quilogramas $(\mathrm{kg})$ dos produtos in natura. No entanto, em relação a $\mathrm{PH}$ evidenciaram apenas $22,1 \%$ do total. Por sua vez o somatório de todos os produtos de origem animal representou $77,9 \%$ da $\mathrm{PH}$, sendo que os cortes de carne bovina foram responsáveis isoladamente por $62,2 \%$ do total desse grupo. 
Figura 1. Quantidade em kg e de PH por grupos de alimentos utilizados em cardápios de RU de Porto Alegre/RS. Setembro/2013.

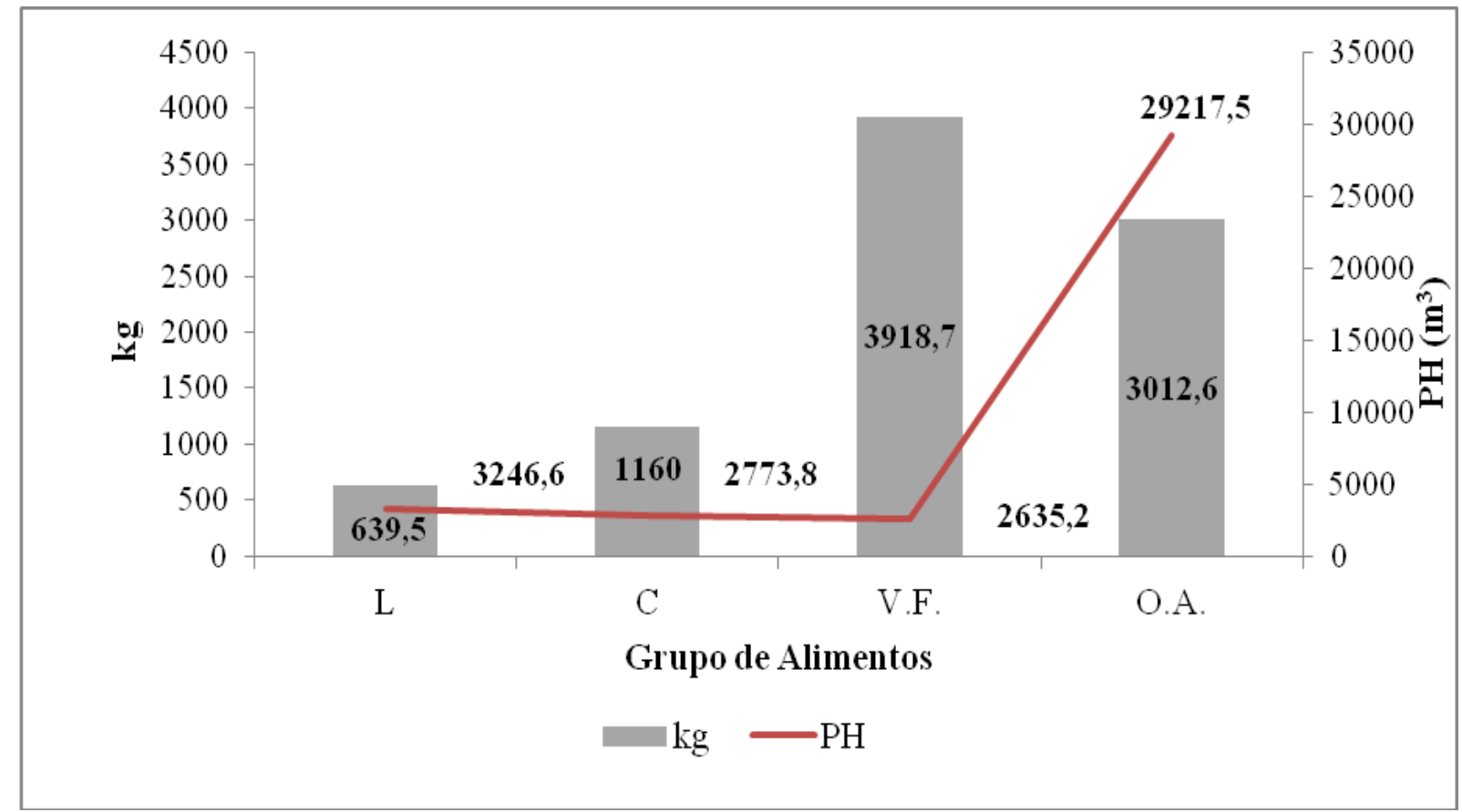

Legenda: kg: quilogramas; $\mathrm{m}^{3}$ : metros cúbicos. L; leguminosas; C: cereal; V.F.: vegetais e frutas; OA: origem animal.

Obs.: os valores dentro de cada coluna de grupo de alimentos estão expressos em kg;

Os valores acima da linha estão expressos $\mathrm{em}^{3}$.

Estudo de Hoekstra e Mekonnen (2012) indicam que a agricultura consome 92\% do total da água, sendo que desse total, $29 \%$ é utilizado direta ou indiretamente para a produção de alimentos para animais. Gerbens-Leenes et al. (2013) apontam que a produção animal é extremamente dispendiosa quanto ao consumo de água em relação ao valor energético nutricional fornecido.

Hoekstra (2011) justifica que esse valor da pegada hídrica se mostra elevado pelo fato de ser considerado no cálculo todo o consumo de água durante a vida incluindo a dessedentação, alimentos, e os processos de higiene ao longo de três anos para a obtenção de $200 \mathrm{~kg}$ de carne desossada, corroborando com os resultados encontrados para a $\mathrm{PH}$ dos produtos de origem animal no cardápio, especialmente o da carne bovina.

Röös et al. (2014) argumentam que os produtos de origem animal são os principais determinantes dos impactos ambientais. No Brasil, Palhares $(2011 ; 2012$; 2014) avaliou processos de $\mathrm{PH}$ na produção de aves e suínos, relatando relação da $\mathrm{PH}$ com a eficiência dos processos de abate dos animais, bem como dos grãos utilizados para alimentação (milho e soja). No caso do frango, a média brasileira da PH foi de $2100 \mathrm{~L} / \mathrm{kg}^{-1}$, inferior ao valor global de $3900 \mathrm{~L} \mathrm{~kg}^{-1}$ (Palhares, 2012).

No estudo de Vanham e Bidoglio (2013) onde foi avaliada a PH de consumo alimentar em países da comunidade europeia os resultados mostraram que as carnes bovina e suína correspondem a 53\% do consumo da pegada hídrica (L/dia). Os cereais representaram 11\% e o grupo dos vegetais outros $9 \%$. Deve ser considerado, no entanto, que a PH da carne suína (4800 $\mathrm{L} \mathrm{kg}^{-1}$ ) consumida em larga escala nos países europeus é bem menor do que a carne bovina (15500 $\mathrm{L} \mathrm{kg}^{-1}$ ) que é, por questões culturais, a principal fonte de consumo animal no estado do RS.

Na proposta de investigação desse trabalho foi realizado o somatório diário de todas as 
preparações produzidas e feito a média per capita diária da $\mathrm{PH}$ considerando a previsão de produção de 1800 refeições. Na Tabela 4 são apresentados os resultados obtidos.

Tabela 4. Média per capita de PH no cardápio diário em restaurante universitário de Porto Alegre/ RS. Setembro/ 2013.

\begin{tabular}{lccc}
\hline \multirow{2}{*}{ Dia } & Semana 1 & & Semana 2 \\
\cline { 2 - 2 } Segunda-feira & 02 a 06 (L) & & 09 a 13 (L) \\
\hline Terça-feira & 2619,8 & & 1184,9 \\
Quarta-feira & 2279,3 & & 2774,0 \\
Quinta-feira & 2996,4 & & 2389,1 \\
Sexta-feira & 1150,6 & & 1080,0 \\
\hline Média & $\mathbf{2 0 6 4 , 0}$ & & $\mathbf{2 1 3 4 , 2}$ \\
\hline DP & $\mathbf{8 1 9 , 1}$ & $\mathbf{9 6 3 , 9}$ \\
\hline
\end{tabular}

Nota: $\mathrm{L}=$ litros.

A PH média por preparação de cardápio ficou em 2099,1 L dia na avaliação da quinzena investigada pelo estudo. No entanto, na avaliação desses mesmos resultados comparados com as preparações do cardápio apontam a média diária de 2717 L na PH para os dias em que o RU serviu a carne bovina (6 dias). Nos dias em que o prato proteico oferecido foi o frango (quatro dias: respectivamente nas segundas e sextas-feiras) identificou-se uma redução de 44,2\% na $\mathrm{PH}$ (1172 L dia) em relação à média geral diária por cardápio. Estudo de Vanham et al. (2013) avaliaram as recomendações de consumo dietético na Europa, utilizando como parâmetro as indicações do ministério da saúde da Alemanha com 2200 calorias. Dos três tipos de dietas avaliadas: saudável, vegetariana e combinada, foi possível constatar que há uma redução da PH conforme a menor presença de produtos de origem animal, principalmente a carne bovina. No Brasil, há pouca repercussão no diz respeito à produção de alimentos ou avaliação de consumo dietético, e no qual se discuta sobre a quantidade de água empregada na produção de alimentos e seu significado em termos nutricionais (Carmo et al., 2007). Estudo de Silva et al. (2013b) avaliou a pegada hídrica de consumidores vegetarianos e não vegetarianos de diferentes níveis de renda familiar em uma cidade do Rio Grande do Norte. No estudo foi apontado que a PH do consumidor vegetariano correspondeu a $58 \% \mathrm{em}$ relação ao consumidor não vegetariano. Situação similar pode ser relacionada aos resultados desse estudo, quando a utilização do frango em relação à carne bovina também mostra valores inferiores. Para Carmo et al. (2007) a culinária é uma das características que mais individualizam as sociedades, em especial quanto a seus pratos típicos. Para Pimentel et al. (2004), o volume de água gasto na produção de alguns tipos de alimentos é muito elevado, e que haveria possibilidades de diminuição significativa da demanda de água a partir de modificações nos itens de consumo nas dietas alimentares de várias populações. Conforme referido no boletim do Water Policy Briefing 25 (IWMI, 2007) se consome entre 2.000 e 5.000 litros de água por dia sem perceber, dependendo do tipo de dieta e cardápio consumido. Ercin e Hoekstra (2014) realizaram um estudo sobre a projeção de cenários futuros da PH do planeta para o ano de 2050. Projetaram quatro cenários considerando variáveis que incluíam o crescimento populacional e econômico; a produção e o padrão de comércio; o padrão de 
consumo e desenvolvimento tecnológico. Os autores destacaram que a redução da $\mathrm{PH}$ da humanidade para níveis considerados sustentáveis é possível mesmo com o aumento populacional, desde que ocorram alterações nos padrões de consumo incluindo mudanças no padrão de alimentação e uso de bioenergia (Ercin e Hoekstra, 2014). No entanto é importante ressaltar que nesse estudo se considera uma situação de particularidade que é o de se avaliar a PH de cardápios elaborados para atender uma coletividade. Diferentemente de projeções individuais onde o consumidor tem o direito de escolha, num restaurante para coletividade os cardápios são previamente elaborados. No Brasil a atribuição pela elaboração dos cardápios cabe ao profissional nutricionista (CFN, 2005). Na produção de refeições, esse profissional, enquanto gestor tem um papel fundamental na avaliação e implementação de rotinas sustentáveis (ADA, 2011; Preuss, 2009), no qual o planejamento de cardápio pode ser incluído. Corroborando com esse enfoque voltado a produção real de refeições para coletividades, estudo realizado na Espanha identificou o segmento relacionado diretamente com a alimentação para coletividade, ou seja, restaurantes, cafés e bares representam $66,74 \%$ do total da $\mathrm{PH}$ no setor de turismo, em grande parte devido à importância de produtos alimentares e bebidas (Cazcarro et al., 2014).De acordo com Aldaya et al. (2010) o uso de recursos para a mensuração da $\mathrm{PH}$ pode promover o desenvolvimento de tecnologias para um uso mais eficiente da água num contexto local de produção e comercialização mais adequado dos produtos. Estes autores descrevem ainda como essenciais o processo de conhecimento das necessidades reais do uso da água para setores como o de alimentos e bebidas, entre outros (Aldaya et al., 2010). Para a American Dietetic Association (ADA) a produção de refeições contempla práticas de conservação dos recursos naturais e de proteção ambiental (Harmon e Gerald, 2007; ADA, 2011). A Green Restaurant Association University (GRAU) avalia em suas auditorias de certificação para restaurantes, além dos aspectos de edificação e do uso dos recursos naturais a questão dos alimentos sustentáveis (GRAU, 2014). Nesse contexto Van Dooren et al. (2014) destaca que o impacto ambiental dos alimentos consumidos deve ser investigado. $\mathrm{Na}$ avaliação das dietas os aspectos nutricionais e de saudabilidade dos alimentos deve ser verificado em relação à sinergia com uma produção ecologicamente sustentável. Como limitação desse estudo relaciona-se o fato de se desenvolver os cálculos considerando o total dos insumos para a produção de 1800 refeições. Não foi possível realizar uma avaliação do consumo individual para cada um dos usuários do serviço, ou ainda de se pesar as sobras e restos dos alimentos que não foram consumidos. Outro aspecto que merece ser mencionado é o da identificação e rastreabilidade dos produtos utilizados para o preparo das refeições. Num contexto de comércio globalizado a utilização dos insumos procede de diferentes regiões, o que nem sempre pode ser identificado na aquisição desses produtos pelos fornecedores, especialmente quando adquiridos em grande quantidade.

\section{CONCLUSÕES}

No fornecimento de refeições para coletividades, não se tem como substituir a utilização de alimentos, no entanto, diferentemente de escolhas individuais ou domésticas, o planejamento de cardápio para coletividade tem uma repercussão direta na questão ambiental. Isso porque as refeições oferecidas contemplam quantidades maiores de insumos para atender a um maior número de beneficiados.

Dessa forma, avaliar a $\mathrm{PH}$ dos insumos utilizados na elaboração de cardápios para coletividades é de fundamental importância para a avaliação dos aspectos da sustentabilidade dos recursos para os quais a água é fundamental. Planejar cardápios de maneira adequada interfere de maneira preventiva no uso dos recursos naturais, bem como pode influenciar positivamente na formação de hábitos e do consumo alimentar mais sustentáveis por parte dos usuários. A realização deste trabalho pretende estimular ainda, a continuidade de pesquisas 
dessa natureza no segmento de produção de refeições.

\section{AGRADECIMENTOS}

À Divisão de Alimentação da Universidade Federal do Rio Grande do Sul e à nutricionista Salete Braga Medeiros pela colaboração no fornecimento de dados. Ao Programa de Pós Graduação em Qualidade Ambiental da Universidade Feevale pelo incentivo e apoio na realização do trabalho.

\section{REFERENCIAS}

ASSOCIAÇÃO BRASILEIRA DAS EMPRESAS DE REFEIÇÕES COLETIVAS - ABERC. Mercado Real. Disponível em: http://www.aberc.com.br/mercadoreal.asp?IDMenu=21 Acesso em: 06 fev. 2015.

AMERICAN DIETETIC ASSOCIATION - ADA. Sustainability in foodservice operations: an update. Journal of the American Dietetic Association, v. 111, n. 9, p. 1286-1294, 2011.

ALDAYA M. M.; HOEKSTRA, A. Y. The water needed for Italians to eat pasta and pizza. Agricultural Systems, v. 103, pp. 351-360, 2010.

http://dx.doi.org/10.1016/j.agsy.2010.03.004

ALDAYA, M. M.; SANTOS, P. M.; LLAMAS, M. R. Incorporating the water footprint and virtual water into policy reflections from the Mancha Occidental region, Spain. Water Resources Management, v. 24, p. 941-958, 2010. http://dx.doi.org/10.1007/s11269009-9480-8

BARTHICHOTO, M.; MATIAS, A. C. G.; SPINELli, M. G. N.; ABREU, E. S. Responsabilidade ambiental: perfil das práticas de sustentabilidade desenvolvidas em unidades produtoras de refeições do bairro de Higienópolis, município de São Paulo. Qualit@s Revista Eletrônica, v.14, n. $1, \quad$ p. $11-12,2013$. http://dx.doi.org/10.18391/qualitas.v14i1.1680

CARMO, R. L. do; OJIMA, A. L. R. O.; OJIMA, R.; NASCIMENTO, T. T. Água virtual, escassez e gestão: O Brasil como grande "exportador" de água. Ambiente \& Sociedade, Campinas, v. 10, n. 2, p. 83-96, 2007. http://dx.doi.org/10.1590/S1414753X2007000200006

CAZCARRO, I; HOEKSTRA, A.Y.; SÁNCHEZ CHÓLIZ, J. The water footprint of tourism in Spain. Tourism Management, v.40, p. 90-101, 2014.

http://dx.doi.org/10.1016/j.tourman.2013.05.010

CONSELHO FEDERAL DE NUTRICIONISTAS - CFN. Resolução CFN no 380, de 9 de dezembro de 2005. Dispõe sobre a definição das áreas de atuação do nutricionista e suas atribuições, estabelece parâmetros numéricos de referência, por área de atuação. Brasília, DF, 2005.

CHAPAGAIN, A. K.; ORR, S. An improved water footprint methodology linking global consumption to local water resources: A case of Spanish tomatoes. Journal of $\begin{array}{llll}\text { Environmental } & \text { Management, } & \text { v.90, } & \text { p.1219-1228, }\end{array}$ http://dx.doi.org/10.1016/j.jenvman.2008.06.006 
COLLARES, L. G. T.; FIGUEIREDO, V. O. Gestão de resíduos sólidos gerados na produção de refeições. Nutrição em Pauta, v. 114, p. 19-24, 2012.

EMPINOTTI, V.; JACOBI, P. R. Novas práticas de governança de água? O uso da pegada hídrica e a transformação das relações entre o setor privado, organizações ambientais e agências internacionais de desenvolvimento. Desenvolvimento e Meio Ambiente, Curitiba, v. 27, p. 23-36, 2013. http://dx.doi.org/10.5380/dma.v27i0.27928

EMPINOTTI, V. L.; TADEU, N. D.; MARTINS, R. S. L. Análise crítica da Pegada Hídrica Cinza na produção de celulose. Revista Ambiente \& Água, Taubaté, v. 8, n. 3, p. 166177, 2013. http://dx.doi.org/10.4136/1980-993X

ERCIN, A. E.; ALDAYA, M. M.; HOEKSTRA, A. Y. Corporate water footprint accounting and impact assessment: the case of the water footprint of sugar-containing carbonated beverage. Water Resources Management, v. 25, p. 721-741, 2011. http://dx.doi.org/10.1007/s11269-010-9723-8

ERCIN, A. E.; HOEKSTRA, A. Y. Water footprint scenarios for 2050: A global analysis. Environment International, v. 64, p. 71-82; 2014. http://dx.doi.org/10.1016/j.envint.2013.11.019

FAGURY, T. Sustentabilidade da lata de aço. Nutrição em Pauta, v. 100, p. 54-57, 2010.

FRANCKE, I. C. M.; CASTRO, J. F. W. Carbon and water footprint analysis of a soap bar produced in Brazil by Natura Cosmetics. Water Resources and Industry, v. 1/2, p. 37 48, 2013. http://dx.doi.org/10.1016/j.wri.2013.03.003

GALLI, A.; WIEDMANN, T.; ERCIN, E.; KNOBLAUCH, D.; EWING, B.; GILJUM, S. Integrating Ecological, Carbon and Water footprint into a "Footprint Family" of indicators: Definition and role in tracking human pressure on the planet. Ecological Indicators, v.16, p.100-112, 2012. http://dx.doi.org/10.1016/j.ecolind.2011.06.017

GERBENS-LEENES, W.; HOEKSTRA, A.Y. The water footprint of sweeteners and bio$\begin{array}{lllll}\text { ethanol. Environment } & \text { International, v.40, p.202-211, }\end{array}$ http://dx.doi.org/10.1016/j.envint.2011.06.006

GERBENS-LEENES, P. W.; MEKONNEN, M. M.; HOEKSTRA, A.Y. The water footprint of poultry, pork and beef: A comparative study in different countries and production systems. Water Resources and Industry, v. 1-2, p. 25-36, 2013. http://dx.doi.org/10.1016/j.wri.2013.03.001

GREEN RESTAURANT ASSOCIATION UNIVERSITY - GRAU. Green restaurant Certification 4.0 Standards. 2014. Disponível em: http://www.dinegreen.com/ restaurants/standards.asp . Acesso em: 22 nov. 2014

HARMON, A. H.; GERALD, B. L. Position of the American Dietetic Association: Food and Nutrition Professionals Can Implement Practices to Conserve Natural Resources and Support Ecological Sustainability. J Am Diet Assoc., v.107, n.6, p.1033-43, 2007. http://dx.doi.org/10.1016/j.jada.2007.04.018

HOEKSTRA, A. Y.; HUNG, P. Q. Virtual water trade: a quantification of virtual water flows between nations in relation to international crop trade. Netherland: UNESCO/IHE, 2002. p. 25-47. (Value of Water Research Series, n. 11). 
HOEKSTRA, A. Y.; CHAPAGAIN, A. K. The water footprints of Morocco and the Netherlands: Global water use as a result of domestic consumption of agricultural commodities. Ecological Economics, v. 64, p.143-151, 2007. http://dx.doi.org/10.1016/j.ecolecon.2007.02.023

HOEKSTRA, A. Y.; CHAPAGAIN, A. K. Globalization of water: sharing the Planet's freshwater resources. Oxford: Blackwell Publishing, 2008. 232p.

HOEKSTRA, A. Y. The water footprint: water in the supply chain. The environmentalist, $n$. 93, p. 12-13, Mar. 2010.

HOEKSTRA, A. Y. How sustainable is Europe's water footprint? Water and Wastewater International, v. 26, n. 2, p. 24-26, 2011.

HOEKSTRA, A. Y.; MEKONNEN, M. M. The water footprint of humanity. Proceedings of the National Academy of Sciences, v. 109, n. 9, p. 3232-3237, 2012. http://dx.doi.org/10.1073/pnas.1109936109

INTERNATIONAL WATER MANAGEMENT INSTITUTE - IWMI. Does food trade save water ? The potential role of food trade in water scarcity mitigation. Colombo, 2007. (IWMI Water Policy Briefing, n. 25). http://dx.doi.org/10.3910/2009.342

MEKONNEN, M. M.; HOEKSTRA, A. Y. The green, blue and grey water footprint of crops and derived crop Products. Hydrology and Earth System Sciences, v. 15, p. 15771600, 2011. http://dx.doi.org/10.5194/hess-15-1577-2011

ORNELAS, L. H. Técnica dietética: seleção e preparo de alimentos. 8. ed. São Paulo: Atheneu, 2007.

PALHARES, J. C. P. Pegada hídrica dos suínos abatidos nos Estados da Região Centro-Sul do Brasil. Acta Scientiarum. Animal Sciences, Maringá, v. 33, n. 3, p. 309-314, 2011. http://dx.doi.org/10.4025/actascianimsci.v33i3.9924

PALHARES, J. C. P. Pegada hídrica das aves abatidas no Brasil na década 2000-2010. In: SEMINÁRIO DE GESTÃO AMBIENTAL NA AGROPECUÁRIA, 3. 25 a 27 abr. 2012, Bento Gonçalves. Trabalhos... Disponível em: http://www.proamb.com.br/downloads/1qsd7a.pdf. Acesso em: 25 ago. 2015.

PALHARES, J. C. P. Pegada hídrica de suínos e o impacto de estratégias nutricionais.

Revista Brasileira de Engenharia Agrícola e Ambiental, v. 18, n. 5, p. 533-538, 2014. http://dx.doi.org/10.1590/S1415-43662014000500010

PEDRO, M. M. R.; CLARO, J. A. C. S. Gestão de perdas em unidade de restaurante popular: um estudo de caso em São Vicente. Qualit@s Revista Eletrônica, v. 19. n. 1, p. 1-10, 2010. http://dx.doi.org/10.18391/qualitas.v9i1.659

PIMENTEL, D.; BERGER, B.; FILIBERTO, D. et al. Water resources: agricultural and environmental issues. BioScience, v. 54, n. 10, p. 909-918, 2004. http://dx.doi.org/10.1641/0006-3568(2004)054[0909:WRAAEI]2.0.CO;2

PREUSS, K. Integrando Nutrição e desenvolvimento sustentável: atribuições e ações do nutricionista. Nutrição em Pauta, n. 99, p. 50-53, 2009. 
RÖÖS, E.; EKELUND, L.; TJÄRNEMO, H. Communicating the environmental impact of meat production: challenges in the development of a Swedish meat guide. Journal of Cleaner Prodution, v. 73, p. 154-164, 2014.

http://dx.doi.org/10.1016/j.jclepro.2013.10.037

SILVA, V. P. R. da; ALEIXO, D. O.; DANTAS NETO, J.; MARACAJÁ, K. F. B.; ARAÚJO, L. E. de. Uma medida de sustentabilidade ambiental: Pegada hídrica. Revista Brasileira de Engenharia Agrícola e Ambiental, v. 17, n. 1, p. 100-105, 2013a. http://dx.doi.org/10.1590/S1415-43662013000100014

SILVA, V. P. R. da; MARACAJÁ, K. F. B.; ARAÚJO, L. E.; DANTAS NETO, J.; ALEIXO, D. O.; CAMPOS, J. H. B. C. Pegada hídrica de indivíduos com diferentes hábitos alimentares. Revista Ambiente \& Água, Taubaté, v. 8, n. 1, p. 250-262, 2013b. http://dx.doi.org/10.4136/ambi-agua.967.

SINISGALLI, P. A.; TADEU, N. D. O uso da pegada hídrica na análise do ambiente. In: JACOBI, P. R.; EMPINOTTI, V. Pegada hídrica: inovação, co-responsabilização e os desafios de sua aplicação. São Paulo: Annablume, 2012. p. 63-82.

SOARES, I. C. C.; SILVA, E. R.; PRIORE, S. E.; RIBEIRO, R. C. L.; PEREIRA, M. M. L. S.; PINHEIRO-SANT'ANA, H. M. Quantificação e análise do custo da sobra limpa em unidades de alimentação e nutrição de uma empresa de grande porte. Revista Nutrição, Campinas, v. 24, n. 4, p. 593-604, 2011.

STRASBURG, V. J.; PASSOS, D. Avaliação do resto per capita de carnes e fatores associados em uma Unidade de Alimentação e Nutrição (UAN). Nutrição em Pauta, v. 22, n. 126, p. 46-50, 2014.

VAN DOOREN, C.; MARINUSSEN, M.; BLONK, H.; AIKING, H.; VELLINGA, P. Exploring dietary guidelines based on ecological and nutritional values: a comparison of six dietary patterns. Food Policy, v. 44, p. 36-46, 2014. http://dx.doi.org/10.1016/j.foodpol.2013.11.002

VANHAM, D.; BIDOGLIO, G. A review on the indicator water footprint for the EU28. Ecological Indicators, v. 26, p. 61-75, 2013. http://dx.doi.org/10.1016/j.ecolind.2012.10.021

VANHAM, D.; MEKONNEN, M.M.; HOEKSTRA, A.Y. The water footprint of the EU for $\begin{array}{lllllll}\text { different diets. Ecological Indicators, v. } & \text { 32, } & \text { p. } & 1-8,\end{array}$ http://dx.doi.org/10.1016/j.ecolind.2013.02.020

YU, Y.; HUBACEK, K.; FENG, K.; GUAN, D. Assessing regional and global water footprints for the UK. Ecological Economics, v. 69, p. 1140-1147, 2010. http://dx.doi.org/10.1016/j.ecolecon.2009.12.008 